\title{
Self-interaction in Green's-function theory of the hydrogen atom
}

\author{
W. Nelson, ${ }^{1, *}$ P. Bokes, ${ }^{2,3}$ Patrick Rinke, ${ }^{3,4}$ and R. W. Godby ${ }^{1,3, \dagger}$ \\ ${ }^{1}$ Department of Physics, University of York, Heslington, York YO10 5DD, United Kingdom \\ ${ }^{2}$ Department of Physics, Faculty of Electrical Engineering and Information Technology, Slovak University of Technology, Ilkovičova 3, \\ 84104 Bratislava, Slovak Republic \\ ${ }^{3}$ European Theoretical Spectroscopy Facility (ETSF) \\ ${ }^{4}$ Fritz-Haber-Institut der Max-Planck-Gesellschaft, Faradayweg 4-6, 14195 Berlin, Germany
}

(Received 5 December 2006; published 14 March 2007)

\begin{abstract}
Atomic hydrogen provides a unique test case for computational electronic structure methods, since its electronic excitation energies are known analytically. With only one electron, hydrogen contains no electronic correlation and is therefore particularly susceptible to spurious self-interaction errors introduced by certain computational methods. In this paper we focus on many-body perturbation theory (MBPT) in Hedin's $G W$ approximation. While the Hartree-Fock and the exact MBPT self-energy are free of self-interaction, the correlation part of the $G W$ self-energy does not have this property. Here we use atomic hydrogen as a benchmark system for $G W$ and show that the self-interaction part of the $G W$ self-energy, while nonzero, is small. The effect of calculating the $G W$ self-energy from exact wave functions and eigenvalues, as distinct from those from the local-density approximation, is also illuminating.
\end{abstract}

DOI: 10.1103/PhysRevA.75.032505

PACS number(s): 31.25.Jf, 31.15.Lc, 31.15.Ar

\section{INTRODUCTION}

Ab initio many-body quantum mechanical calculations are crucially important to our understanding of the behavior of atomic, molecular, and condensed matter systems. It is wellknown that predicting the behavior of these systems requires the description of electronic correlation. While densityfunctional theory (DFT) in the local-density approximation (LDA) does this with startling success in many cases, it does so at the expense of a nonphysical electron self-interaction. For delocalized electron systems this self-interaction becomes negligible, but in atomic or strongly localized electronic systems it plays an important role. If one is interested in the calculation of quasiparticle excitation spectra, manybody perturbation theory (MBPT) is formally a correct way to proceed. For solids, MBPT in Hedin's $G W$ approximation [1] has become the method of choice, but it is also increasingly being applied to molecular systems and clusters. The $G W$ self-energy can be decomposed into correlation and exchange parts, where the latter is the same as the Fock operator encountered in Hartree-Fock theory and thus selfinteraction free. While the exact self-energy must also be free of self-interaction, the correlation part of the $G W$ selfenergy does not have this property. To investigate the influence of self-interaction in the $G W$ approach the hydrogen atom provides an ideal case because the exact solution is known analytically.

Hydrogen in its solid phase has previously been studied within the $G W$ approximation by Li et al. [2], who analyzed the transition between the high-pressure solid phase and the low density, atomiclike limit. For individual atoms, $G W$ electron removal and addition energies (we use the term "quasiparticle" energies by analogy with the solid-state situation)

\footnotetext{
*Present address: Department of Physics, King's College London, Strand, London WC2R 2LS, United Kingdom.

${ }^{\dagger}$ Electronic address: rwg3@york.ac.uk
}

have been investigated by Shirley and Martin [3], Dahlen et al. [4,5], Stan et al. [6], and Delaney et al. [7], although hydrogen was not considered. These studies have shown that $G W$, in general, gives quasiparticle properties which are much improved over DFT and Hartree-Fock methods, even for atoms.

In this paper we use the hydrogen atom as a benchmark system to quantify the self-interaction error in the $G W$ approach. Since the self-energy diagrams beyond $G W$, known as the vertex correction, must by definition correct this selfinteraction error, our findings are relevant for research into vertex functions for the many-electron problem.

Attention has recently focused on the prospects for improving the usual non-self-consistent $G W$ calculations by choosing an initial Green's function, $G_{0}$, that is physically more reasonable than the LDA (e.g., $[2,8,9])$. We explore this here by determining the sensitivity of the self-interaction error to the use of the exact hydrogenic orbitals and energies in place of those from the local-density approximation (LDA). We also assess the error introduced into $G W$ calculations by employing first-order perturbation theory in solving the quasiparticle equation (as opposed to the full numerical solution), and we analyze the quasiparticle wave functions that emerge from a full solution.

\section{HARTREE-FOCK VERSUS DFT-LDA}

In many-body perturbation theory the quasiparticle excitation energies $\epsilon_{i \sigma}$ and wave functions $\psi_{i \sigma}$ are the solutions of the quasiparticle equation

$$
H_{0}(\mathbf{r}) \psi_{i \sigma}(\mathbf{r})+\sum_{\sigma^{\prime}} \int d \mathbf{r}^{\prime} M_{\sigma \sigma^{\prime}}\left(\mathbf{r}, \mathbf{r}^{\prime} ; \epsilon_{i \sigma}^{\mathrm{qp}}\right) \psi_{i \sigma^{\prime}}\left(\mathbf{r}^{\prime}\right)=\epsilon_{i \sigma}^{\mathrm{qp}} \psi_{i \sigma}(\mathbf{r})
$$

where, in Hartree atomic units, $H_{0}(\mathbf{r})=-\frac{1}{2} \nabla^{2}+v_{e x t}(\mathbf{r})$ and $v_{\text {ext }}(\mathbf{r})$ is the external potential. It is customary to divide the 
mass operator $M$ into the local Hartree potential $\left(v_{H}\right)$ and the nonlocal self-energy $(\Sigma)$

$$
M_{\sigma \sigma^{\prime}}\left(\mathbf{r}, \mathbf{r}^{\prime} ; \boldsymbol{\epsilon}\right)=v_{H}(\mathbf{r}) \delta\left(\mathbf{r}-\mathbf{r}^{\prime}\right) \delta_{\sigma \sigma^{\prime}}+\Sigma_{\sigma \sigma^{\prime}}\left(\mathbf{r}, \mathbf{r}^{\prime} ; \boldsymbol{\epsilon}\right) .
$$

Omitting correlation contributions from $\Sigma$ yields the exactexchange or Hartree-Fock case, where the self-energy takes the form

$$
\sum_{\sigma \sigma^{\prime}}^{x}\left(\mathbf{r}, \mathbf{r}^{\prime}\right)=-\sum_{i}^{o c c} \frac{\psi_{i \sigma}(\mathbf{r}) \psi_{i \sigma}^{*}\left(\mathbf{r}^{\prime}\right)}{\left|\mathbf{r}-\mathbf{r}^{\prime}\right|} \delta_{\sigma \sigma^{\prime}}
$$

and the Hartree potential is given by

$$
v_{H}(\mathbf{r})=\sum_{i, \sigma}^{o c c} \int d \mathbf{r}^{\prime} \frac{\psi_{i \sigma}\left(\mathbf{r}^{\prime}\right) \psi_{i \sigma}^{*}\left(\mathbf{r}^{\prime}\right)}{\left|\mathbf{r}-\mathbf{r}^{\prime}\right|} .
$$

Since the sum runs over all occupied states the Hartree potential contains an artificial interaction of electrons with themselves. For the hydrogen atom this so-called selfinteraction of the electron is the only content of the Hartree potential and may be calculated analytically using the exact ground-state wave function

$$
\psi_{1 s}^{\uparrow}(r)=\frac{1}{\sqrt{\pi}} e^{-r}
$$

as

$$
v_{H}(r)=\frac{1}{r}\left\{1-(1+r) e^{-2 r}\right\} .
$$

The self-interaction is a positive, decreasing function and hence tends to delocalize the wave function (i.e., incorrectly pushes its weight away from the nucleus).

In Hartree-Fock the self-interaction terms introduced in the Hartree potential are exactly canceled by the Fock operator $\Sigma_{x}$. This makes Hartree-Fock exact for one-electron systems such as the hydrogen atom. However, the lack of correlation renders Hartree-Fock unsuitable for many polyatomic systems of interest.

Of more practical use is Kohn-Sham [10] densityfunctional theory, which by virtue of the Hohenberg-Kohn theorem [11] establishes an exact and universal functional relationship between the ground-state density $n(\mathbf{r})$ and the total energy $E$ of a system. Mapping the system of interacting electrons onto a fictitious system of noninteracting electrons, that reproduces the exact density, yields the KohnSham equations:

$$
\left[H_{0}(\mathbf{r})+v_{H}(\mathbf{r})+v_{x c}(\mathbf{r})\right] \phi_{i \sigma}(\mathbf{r})=\epsilon_{i \sigma}^{\mathrm{KS}} \phi_{i \sigma}(\mathbf{r}) .
$$

All electron-electron interactions beyond the Hartree mean field are encompassed by the exchange-correlation potential $v_{x c}$, which is formally given as the functional derivative of the exchange-correlation energy $E_{x c}$ :

$$
v_{x c}(\mathbf{r})=\frac{\delta E_{x c}[n]}{\delta n(\mathbf{r})} .
$$

In analogy to the quasiparticle equation (1) the Kohn-Sham eigenvalues $\epsilon_{i \sigma}^{\mathrm{KS}}$ are often interpreted as excitation energies, although this is not formally justified.
One of the most common approximations for $E_{x c}$ is the local-density approximation (LDA) [10], in which the manybody exchange and correlation contributions to the total energy $\left(E_{x c}\right)$ are included by comparison with the homogeneous electron gas (HEG):

$$
E_{x c}^{\mathrm{LDA}}[n]=\int d \mathbf{r} n(\mathbf{r}) \epsilon_{x c}^{\mathrm{HEG}}[n(\mathbf{r})] .
$$

Here [12] we follow the parametrization of Perdew and Zunger [13] for the exchange-correlation energy density $\epsilon_{x c}^{\mathrm{HEG}}[n(\mathbf{r})]$ of the homogeneous electron gas based on the data of Ceperley and Alder [14].

The LDA has been remarkably successful at accounting for correlation even in systems that are highly inhomogeneous. However, it is well-known that the introduction of correlation in the LDA comes at the expense of the exact treatment of the self-interaction. Because the exchange functional is taken from the homogeneous electron gas it no longer cancels the spurious self-interaction present in the Hartree term. In most systems this is a minor effect and is more than compensated by the improved treatment of the electron correlation. The LDA can be improved by explicitly removing the self-interaction [13], however, this becomes increasingly difficult as the system's complexity is increased.

\section{THE $G W$ APPROXIMATION}

In Hedin's $G W$ approximation [1] the self-energy in Eq. (2) is given by

$$
\Sigma_{\sigma \sigma}\left(\mathbf{r}, \mathbf{r}^{\prime} ; \epsilon\right)=\frac{i}{2 \pi} \int_{-\infty}^{\infty} d \epsilon^{\prime} e^{i \epsilon^{\prime} \delta} G_{\sigma \sigma}\left(\mathbf{r}, \mathbf{r}^{\prime} ; \epsilon+\epsilon^{\prime}\right) W\left(\mathbf{r}, \mathbf{r}^{\prime} ; \epsilon\right),
$$

where $\delta$ is an infinitesimal positive time. At the level of $G W$, spin flips are not accounted for [2], and the input Green's function is diagonal in its spin representation $G_{\sigma \sigma}$ $=G_{\sigma \sigma^{\prime}} \delta_{\sigma \sigma^{\prime}}$.

In common with usual $G W$ calculations, a Kohn-Sham Green's function $G^{0}$ is used for $G$, given by [15]

$$
G_{\sigma \sigma}^{0}\left(\mathbf{r}, \mathbf{r}^{\prime}, \epsilon\right)=\sum_{i} \frac{\phi_{i \sigma}(\mathbf{r}) \phi_{i \sigma}^{*}\left(\mathbf{r}^{\prime}\right)}{\epsilon-\epsilon_{i \sigma}^{\mathrm{KS}} \mp i \delta},
$$

and makes the noninteracting polarizability

$$
\chi_{\sigma \sigma}^{0}\left(\mathbf{r}, \mathbf{r}^{\prime}, \epsilon\right)=-\frac{i}{\pi} \int_{-\infty}^{\infty} d \epsilon^{\prime} G_{\sigma \sigma}^{0}\left(\mathbf{r}, \mathbf{r}^{\prime} ; \epsilon^{\prime}-\epsilon\right) G_{\sigma \sigma}^{0}\left(\mathbf{r}^{\prime}, \mathbf{r} ; \epsilon^{\prime}\right)
$$

and the dielectric function spin-dependent (though spindiagonal). The inverse dielectric function in the randomphase approximation

$$
\varepsilon^{-1}\left(\mathbf{r}, \mathbf{r}^{\prime}, \boldsymbol{\epsilon}\right)=\left[\delta\left(\mathbf{r}-\mathbf{r}^{\prime}\right)-\int d \mathbf{r}^{\prime \prime} v\left(\mathbf{r}, \mathbf{r}^{\prime \prime}\right) \sum_{\sigma} \chi_{\sigma \sigma}^{0}\left(\mathbf{r}^{\prime \prime}, \mathbf{r}^{\prime}, \boldsymbol{\epsilon}\right)\right]^{-1}
$$

and thus the screened Coulomb interaction 


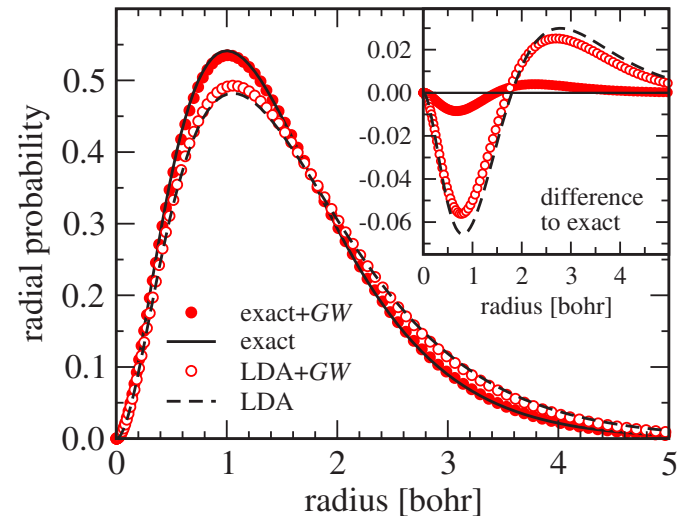

FIG. 1. (Color online) Radial probability distributions of the hydrogen $1 s$ state: the quasiparticle wave function deviates only slightly from the exact wave function, when the latter is used as a starting point $(\operatorname{exact}+G W)$. The LDA wave function, on the other hand, is more delocalized as a result of the inherent self-interaction. Adding quasiparticle corrections (LDA $+G W)$ brings the resulting quasiparticle wave function slightly closer to the exact one again. The inset shows the difference to the exact wave function.

$$
W_{0}\left(\mathbf{r}, \mathbf{r}^{\prime}, \epsilon\right)=\int d \mathbf{r}^{\prime \prime} \varepsilon^{-1}\left(\mathbf{r}, \mathbf{r}^{\prime \prime}, \epsilon\right) v\left(\mathbf{r}^{\prime \prime}, \mathbf{r}^{\prime}\right)
$$

then emerge as spin-independent quantities, giving rise to the simple spin dependence in the $G W$ self-energy [Eq. (10)].

For numerical convenience and physical insight we separate the $G W$ self-energy (10) according to

$$
\Sigma_{\sigma \sigma}=-i\left[G_{\sigma \sigma} v+G_{\sigma \sigma}(W-v)\right]=\Sigma_{\sigma \sigma}^{x}+\Sigma_{\sigma \sigma}^{c} .
$$

The first term $\left(\sum_{\sigma \sigma}^{x}\right)$ corresponds to the Fock operator in Eq. (3) and will exactly cancel the self-interaction introduced by the Hartree potential. It is therefore immediately clear that any deviation from the exact result for hydrogen can only come from the correlation part of the self-energy $\left(\Sigma_{\sigma \sigma}^{c}\right)$.

The incorrect self-interaction affects the electron removal energies (here, the ionization potential). For electron addition energies such as the electron affinity, the entire Hartree potential has a physically reasonable interpretation, since it acts on the wave function of the originally unoccupied state which has not contributed to the electron density.

\section{COMPUTATIONAL APPROACH}

We solve the quasiparticle equation (1) with the $G_{0} W_{0}$ self-energy (10) for the quasiparticle energies and wave functions by fully diagonalizing the quasiparticle Hamiltonian in the basis of the single particle orbitals of the noninteracting system. Since the ground state of the hydrogen atom (5) is spherically symmetric, it is sufficient to describe all non-local operators in the $G W$ formalism by two radial and one spin coordinates, $r, r^{\prime}$, and $\sigma$ and one angular coordinate, $\theta$, that denotes the angle between the vectors $\mathbf{r}$ and $\mathbf{r}^{\prime}$. The self-energy (10) then assumes the much simpler form
TABLE I. Quasiparticle energies (eV) for the $1 s$ state of hydrogen (the ionization potential) obtained by diagonalizing the quasiparticle Hamiltonian (1). Two $G W$ calculations are shown, starting from the LDA and from exact Kohn-Sham, respectively. For comparison, the Hartree-Fock (HF) and LDA eigenvalues are also shown.

\begin{tabular}{ccccc}
\hline \hline Exact & HF & LDA & LDA $+G W$ & Exact $+G W$ \\
\hline-13.61 & -13.61 & -6.36 & -12.66 & -13.40 \\
\hline \hline & & & \\
& $\Sigma_{\sigma \sigma}\left(r, r^{\prime}, \theta ; \omega\right)=\sum_{l=0}^{\infty}\left[\Sigma_{l \sigma}\left(r, r^{\prime} ; \omega\right)\right] P_{l}(\cos \theta) \delta_{\sigma, \sigma}$, & $(16)$
\end{tabular}

where $P_{l}(\cos \theta)$ is a Legendre polynomial of order $l$.

The Legendre expansion coefficients of the self-energy are calculated directly, thereby surpassing the need for a numerical treatment of the angular dependence. We use a realspace and imaginary time representation [16] to calculate the self-energy from the noninteracting Green's function $G_{0}$. The expression for the self-energy on the real frequency axis is obtained by analytic continuation [16]. The current implementation has been successfully applied to jellium clusters [17] and light atoms [7].

Our code allows us to solve the quasiparticle equation (1) for the $G W$ self-energy with no further approximation. However, in order to separate the contribution that arises from the correlation part of the self-energy from that of the exchange part and the Hartree and exchange-correlation potential we also solve the quasiparticle equation with the frequently made approximation that the quasiparticle wave functions are given by the Kohn-Sham wave functions. The resulting equation for the quasiparticle energies is

$$
\epsilon_{i \sigma}^{q p}=\epsilon_{i \sigma}^{\mathrm{KS}}+\left\langle\Sigma_{\sigma \sigma}^{x}\right\rangle+\left\langle\Sigma_{\sigma \sigma}^{c}\left(\epsilon_{i \sigma}^{q p}\right)\right\rangle-\left\langle v_{\sigma}^{x c}\right\rangle,
$$

where the brackets \langle\rangle denote matrix elements with respect to the Kohn-Sham wave function $\phi_{i \sigma}$.

In order to explore the role of the starting points for a $G W$ calculation, two possible Kohn-Sham input Green's functions are chosen. First, the familiar LDA, and, second, the exact Kohn-Sham solution for the hydrogen atom which has the exact wave function of the hydrogen $1 s$ state (5) and $v_{x c}(\mathbf{r})=-v_{H}(\mathbf{r})$. (This exact Kohn-Sham Green's function, incidentally, differs from the exact Green's function of the hydrogen atom because the exact Kohn-Sham unoccupied eigenvalues do not signify electron affinities. The exact Green's function cannot be constructed from any orthonormal set of one-particle wave functions.)

\section{RESULTS AND DISCUSSION}

The calculated ionization potentials (from a full solution of the quasiparticle equation) are shown in Table I. The selfinteraction errors in the two $G W$ quasiparticle energies are seen to be fairly small: $0.95 \mathrm{eV}$ when the approximate LDA Kohn-Sham starting point is used, and the much smaller $0.21 \mathrm{eV}$ when the exact Kohn-Sham starting point is used. Clearly the LDA is such a physically poor representation of 
the correct physics in this extreme system (owing to the large self-interaction present in the LDA calculation itself, as reflected in the large error in the LDA Kohn-Sham eigenvalue) that it forms a very unsuitable starting point for $G W$. However, a physically reasonable starting point reduces the $G W$ self-interaction error to a small size.

Since $\Sigma_{c}$ gives a nonvanishing contribution to the hydrogen $1 s$ state, even if the analytic solution is used as a starting point, the quasiparticle wave function will differ from the exact one. Figure 1 shows that the $G W$ correlation gives rise to a slight delocalization of the quasiparticle wave function in this case. This relaxation, however, now makes the quasiparticle wave function an eigenfunction of the quasiparticle Hamiltonian. In the LDA the self-interaction error is much more pronounced and the wave function becomes significantly more delocalized. The $G W$ self-energy corrects this to a small extent (as reflected in the quasiparticle wave function), but the remaining discrepancy reiterates the unsuitability of the LDA as a starting point for $G W$ in this selfinteraction-dominated atom.

For an analysis of the contributions to the self-energy we turn to the perturbative solution of the quasiparticle equation using Eq. (17), shown in Table II. When the exact KohnSham wave function and eigenvalues are used, as in the Hartree-Fock case the exchange part of the self-energy is seen to cancel the self-interaction contribution from the Hartree potential exactly. The correlation part, on the other hand, is not zero, but amounts to a self-polarization of $0.25 \mathrm{eV}$. When the LDA is used as the starting point the influence of the LDA wave function on the exchange operator becomes apparent and it reduces from $-17.00 \mathrm{eV}$ in the exact case to $-15.38 \mathrm{eV}$. This corrects the highly overestimated LDA eigenvalue for the $1 s$ state of $-6.36 \mathrm{eV}$ (see Table I) to $-13.49 \mathrm{eV}$. However, in this case the contribution from the correlation part of the $G W$ self-energy is even larger than when starting from the exact case and increases the quasiparticle energy to $-12.93 \mathrm{eV}$.
TABLE II. Quasiparticle energies (eV) for the $1 s$ state of hydrogen obtained by solving Eq. (17). The contributions from the exchange $\left\langle\Sigma_{x}\right\rangle$ and correlation $\left\langle\Sigma_{c}\right\rangle$ part of the self-energy are compared to that of the exchange-correlation potential $\left\langle v_{x c}\right\rangle$ for the LDA and the exact case $\left(v_{x c}=-v_{H}\right)$ as a starting point. Exact value for $\epsilon_{1 s}$ is $-13.61 \mathrm{eV}$.

\begin{tabular}{lcccc}
\hline \hline Kohn-Sham $G_{0}$ & $\epsilon_{1 s}^{G W}$ & $\left\langle\Sigma_{x}\right\rangle$ & $\left\langle v_{x c}\right\rangle$ & $\left\langle\Sigma_{c}\right\rangle$ \\
\hline LDA & -12.93 & -15.38 & -8.25 & 0.56 \\
Exact & -13.35 & -17.00 & -17.00 & 0.25 \\
\hline \hline
\end{tabular}

\section{CONCLUSION}

We have performed spin-resolved benchmark calculations for the $G W$ formalism using the analytically known solutions of the hydrogen atom as a reference, making the selfinteraction error introduced by the correlation part of the $G W$ self-energy directly assessable. When the exact Kohn-Sham Green's function is used as the input to $G W$, the selfinteraction error is small $(0.21 \mathrm{eV}, 1 / 30$ the size of that in the LDA), but not negligible. If the LDA Kohn-Sham Green's function is used, as done in many $G W$ calculations for more complex systems, a larger self-interaction error remains, inherited from the LDA starting point.

\section{ACKNOWLEDGMENTS}

This work was supported by the NATO Security Through Science Programme (EAP.RIG.981521), the Slovak grant agency VEGA (Project No. 1/2020/05), and the EU's 6th Framework Programme through the NANOQUANTA Network of Excellence (NMP4-CT-2004-500198).
[1] L. Hedin, Phys. Rev. 139, A796 (1965).

[2] J.-L. Li, G.-M. Rignanese, E. K. Chang, X. Blase, and S. G. Louie, Phys. Rev. B 66, 035102 (2002).

[3] E. L. Shirley and R. M. Martin, Phys. Rev. B 47, 15404 (1993).

[4] N. E. Dahlen and U. von Barth, Phys. Rev. B 69, 195102 (2004).

[5] N. E. Dahlen and R. van Leeuwen, J. Chem. Phys. 122, 164102 (2005).

[6] A. Stan, N. E. Dahlen, and R. van Leeuwen, Europhys. Lett. 76, 298 (2006).

[7] K. Delaney, P. García-González, A. Rubio, P. Rinke, and R. W. Godby, Phys. Rev. Lett. 93, 249701 (2004).

[8] P. Rinke, A. Qteish, J. Neugebauer, C. Freysoldt, and M. Scheffler, New J. Phys. 7, 126 (2005).

[9] J.-L. Li, G.-M. Rignanese, and S. G. Louie, Phys. Rev. B 71, 193102 (2005).
[10] W. Kohn and K. J. Sham, Phys. Rev. 140, A1133 (1965).

[11] P. Hohenberg and W. Kohn, Phys. Rev. 136, B864 (1964).

[12] A local spin density calculation improves the eigenenergy of the hydrogen $1 s$ only slightly [18].

[13] J. P. Perdew and A. Zunger, Phys. Rev. B 23, 5048 (1981).

[14] D. M. Ceperley and B. J. Alder, Phys. Rev. Lett. 45, 566 (1980).

[15] The minus (plus) sign in the denominator applies to occupied (unoccupied) states.

[16] H. N. Rojas, R. W. Godby, and R. J. Needs, Phys. Rev. Lett. 74, 1827 (1995).

[17] P. Rinke, K. Delaney, P. García-González, and R. W. Godby, Phys. Rev. A 70, 063201 (2004).

[18] S. Kotochigova, Z. H. Levine, E. L. Shirley, M. D. Stiles, and C. W. Clark, Phys. Rev. A 55, 191 (1997); 56, 5191 (1997); http://physics.nist.govPhysRefData/DFTdata/ 